\title{
Stiffness, Strength and Interwall Sliding in Aligned and Continuous Multi-walled Carbon Nanotube/Glass Composite Microcantilevers
}

Geoffrey Otieno, Antal Koos ${ }^{+}$, Frank Dillon, Noor Azlin Yahya, Claire E J Dancer, Gareth M. Hughes, Nicole Grobert and Richard I Todd*

Department of Materials, University of Oxford, Oxford, OX1 3PH, UK

*Corresponding author. Fax: +44 (0)1865 273789. E-mail: richard.todd@materials.ox.ac.uk

${ }^{+}$Present address: Institute for Technical Physics and Materials Science, Centre for Energy Research, Konkoly-Thege ut 29-33, Budapest, 1121, Hungary

Keywords: carbon nanotubes; glass matrix composites; mechanical properties; microcantilevers

\section{Abstract}

Individual perfect multi-wall carbon nanotubes (MWCNT) can exhibit exceptional properties, such as an elastic modulus of $\sim 1$ TPa. However, integration of carbon nanotubes (CNTs) in bulk ceramic composites has not yet resulted in the significant improvements in mechanical properties that such data suggest to be achievable. Composites with aligned and continuous CNTs and with high CNT volume fractions might be expected to maximise the improvements. We have produced aligned MWCNT preforms by chemical vapour deposition and fabricated dense, aligned and continuous 20\% MWCNT/glass composites. This was achieved by infiltration of a ceramic precursor sol into the interstices of a MWCNT preform and consolidation by hot-pressing. The elastic modulus was measured using microcantilever tests and showed a 32\% improvement over that of glass. The Young's modulus inferred for the MWCNTs in the composite was $200 \pm 20$ GPa. The load-displacement curves showed a nonlinear and hysteretic behaviour which was attributed to interwall sliding within the MWCNTs. Apparent bridging of the cracks by the MWCNTs and a load maximum preceding failure 
were observed in the composite, indicating progressive toughening with crack growth. The results are discussed in terms of the microstructures of the MWCNTs and composites.

\section{Introduction}

There have been many attempts during the last 20 years to take advantage of the excellent properties offered by individual carbon nanotubes (CNT) by incorporating them in composites [1]. With glass or ceramic matrices, improvements in properties such as wear resistance [2,3] and electrical and thermal conductivity [4,5] have been reported but most attention has been focused on mechanical properties. Most studies have investigated randomly orientated CNTs within matrix [6,7] but these composites have shown only modest improvements in stiffness and toughness [8,9]. From the experience with advanced fibre composites, aligning the CNTs would be expected to result in enhanced mechanical properties in the direction of alignment. It should also enable higher volume fractions of CNTs to be incorporated.

Two general methods of fabricating aligned CNT ceramic composites have been reported: i) alignment of CNTs during composite processing e.g. by extrusion [10] or electrophoretic deposition [11,12] and ii) infiltration of an aligned CNT preform with a ceramic precursor $[4,13]$. The first method suffers from the susceptibility of CNTs to agglomeration during processing and the CNTs are not continuous within the final composite. The second method (infiltration) results not only in aligned CNTs, but also the CNTs are continuous within the matrix. We have previously reported the use of aligned CNT preforms for infiltration with a sol precursor [4] while Chandrashekar et al. [13] used chemical vapour infiltration (CVI). Both of these routes produced porous composites, however, and their mechanical properties have not been investigated. 
In the present study, dense glass matrix composites containing continuous and aligned multiwalled CNTs (MWCNTs) have been produced based on our previous infiltration-based route [4] but using hot pressing rather than pressureless sintering during densification. The alignment allows significantly higher volume fractions of MWCNTs to be incorporated than has been achieved with unaligned composites. The microstructure, elastic properties and fracture behaviour of the composites are reported. The mechanical properties are investigated using microcantilever beams loaded using a nanoindenter. This recently developed technique has two significant advantages in the present case. Firstly, it enables the limited volumes of material available with present processing technology to be assessed before expending the considerable resources required to scale up production of these complex and expensive materials. This extends a previous approach using conventional nanoindentation of surfaces to characterise very limited amounts of ordered ceramic/CNT composites [14]. Secondly, microcantilever beams enable testing on the scale of the microstructure, enabling fundamental processes such as interwall sliding which underpin the macroscopic behaviour, to be studied in isolation, thus leading to a deeper understanding of the factors ultimately responsible for the mechanical properties of such composites.

\section{Experimental}

\subsection{Synthesis of aligned MWCNT preforms}

Aligned MWCNTs were grown on quartz substrates $\left(10 \times 20 \mathrm{~mm}^{2}\right)$ using an aerosol CVD setup consisting of a piezoelectric generator, a quartz tube $(2.2 \mathrm{~cm}$ inner diameter), a $50 \mathrm{~cm}$ long horizontal tube furnace, gas flow controller and acetone gas trap [15]. A solution of $5 \mathrm{wt}$ $\%$ ferrocene $\left(\mathrm{Fe}\left[\mathrm{C}_{5} \mathrm{H}_{5}\right]_{2}\right.$, Aldrich $98 \%$, the iron catalyst precursor) in toluene $\left(\left[\mathrm{C}_{6} \mathrm{H}_{5} \mathrm{CH}_{3}\right]\right.$, Aldrich, the carbon source) was used for the synthesis of aligned MWCNTs. The synthesis temperature was $800{ }^{\circ} \mathrm{C}$ with Ar as carrier gas at a flow rate of $1.7 \times 10^{-5} \mathrm{~m}^{3} / \mathrm{s}$. The resulting 
nanotubes could be mechanically stripped from the substrate to produce freestanding preforms of aligned MWCNTs with length 3-5 mm.

\section{$2.2 \quad$ Preparation of the sol}

The sol was prepared using a method we have reported previously [4,8]. Briefly, an initial solution was prepared by mixing $4.7 \mathrm{ml}$ tetraethyl orthosilicate $\left(\mathrm{Si}\left[\mathrm{OC}_{2} \mathrm{H}_{5}\right]_{2}\right.$, Aldrich $\left.>98 \%\right)$ with $2.25 \mathrm{ml}$ of $4 \mathrm{M}$ aluminium trisecbutoxide $\left(\mathrm{Al}\left[\mathrm{OCH}\left(\mathrm{CH}_{3}\right) \mathrm{C}_{2} \mathrm{H}_{5}\right]_{3}\right.$, Aldrich, $\left.>98 \%\right)$. A second solution was prepared by mixing $4.7 \mathrm{ml}$ ethanol with $1 \mathrm{ml}$ of $2 \mathrm{M}$ sodium acetate $\left(\mathrm{NaO}_{2} \mathrm{CH}_{3}, \mathrm{BDH}, \mathrm{UK}, 99 \%\right), 0.7 \mathrm{ml}$ of trimethyl borate $\left(\mathrm{B}\left[\mathrm{OCH}_{3}\right]_{3}\right.$, Aldrich, $\left.>98 \%\right), 0.5 \mathrm{ml}$ $67 \%$ nitric acid (Aldrich) and $0.5 \mathrm{ml}$ of distilled water. The two solutions were mixed to produce a sol that would yield aluminoborosilicate glass of $63 \mathrm{wt} \% \mathrm{SiO}_{2}, 24 \mathrm{wt} \% \mathrm{Al}_{2} \mathrm{O}_{3}, 10$ wt $\% \mathrm{~B}_{2} \mathrm{O}_{3}$ and 3 wt $\% \mathrm{Na}_{2} \mathrm{O}$.

\subsection{Infiltration of the MWCNT preforms with ABS sol and hot-pressing}

Vacuum infiltration was used to introduce the sol into the interstices of the aligned MWCNT preforms. The sol was left to gel and dry within the interstices of the aligned MWCNT preforms at room temperature for 12 hours. The gel infiltrated aligned MWCNT performs were further dried at $350{ }^{\circ} \mathrm{C}$ in air for 3 hours to remove unreacted organic material. Five infiltrations were performed to increase the amount of matrix precursor introduced in the interstices of the preform. The infiltrated and dried aligned MWCNT preforms were loaded typically 3 at a time directly in a $15 \mathrm{~mm}$ diameter graphite die and the gaps between the preforms were then filled with calcined glass precursor. These compacts were then hot pressed under Ar. The loading of the preforms was such that the pressure was applied normal to the direction of alignment of the MWCNT as shown schematically in Figure 1 so that 
densification could take place in the pressing direction without excessive bending of the CNTs.

The hot-pressing conditions were: pressure $25 \mathrm{MPa}$, peak temperature $1100{ }^{\circ} \mathrm{C}$, heating rate $25{ }^{\circ} \mathrm{C} / \mathrm{min}$, holding time at peak temperature $10 \mathrm{~min}$ and natural cooling rate. After hot pressing, regions containing a single aligned MWCNT preform were identified and excised by diamond sawing and grinding to produce specimens with MWCNT aligned over thicknesses of several mm. For comparison, pure ABS glass was also produced by hot pressing under the same conditions.

\subsection{Analysis}

The densities of the hot-pressed composite specimens were measured according to the Archimedes method with deionized water as the immersion medium. For the MWCNT preforms, the density was obtained from the measured mass and dimensions. Optical microscopy, scanning electron microscopy (SEM, JEOL 6500F) and transmission electron microscopy (TEM, JEOL 2000FX, $200 \mathrm{kV}$ and JEOL 4000, $400 \mathrm{kV}$ ) were used to examine the microstructures of the composites. Both the unreinforced glass and the composites were polished using diamond slurries from $25 \mu \mathrm{m}$ to $1 \mu \mathrm{m}$ before microstructural studies using SEM. Also, focused ion beam (FIB) milling was used to produce polished cross-sectional surfaces (FEI FIB200, $30 \mathrm{kV}$ ) and to perform FIB tomography (Zeiss NVision 40, $30 \mathrm{kV}$ ). The tomographic reconstruction was done using Avizo software (Mercury computer systems, France).

TEM specimens were produced by in-situ FIB lift-out and transferred to a copper grid. To measure the elastic properties and strength, FIB milling was used to produce micro-cantilever beams of triangular cross-section in the bulk composite and on unreinforced glass. The length of the cantilever was in the alignment direction of the MWCNTs in the composite specimens. 
Microcantilever bend tests were performed on an MTS XP nanoindenter fitted with a piezoelectric stage that allows constant force scanning mode images to be obtained in order to position the load point accurately on the specimen. The loading of the microcantilever beam was done by positioning the indenter tip at a location along the centre line of the top face of the beam, near the free end and applying displacement at a rate of $20 \mathrm{~nm} / \mathrm{s}$. More details of the basic technique are given in [16]. Five microcantilever beams were tested for the ABS glass and five for the CNT/ABS composite. Fractography was carried out using the SEM.

\section{Results and Analysis}

\subsection{Microstructural Characterisation}

Inspection of the polished sections of the specimens showed that although the CNT preforms had been broken up by the hot pressing, there were many crack-free and undistorted regions with dimensions of several hundred micrometres in which to investigate the properties using microcantilevers tests.

Figure $2 \mathrm{a}$ and $\mathrm{b}$ show SEM images of FIB polished cross-sections normal and parallel to the alignment direction of the CNTs. The bright rounded/oval features in the normal crosssection (Fig. 2a) are the CNT cross sections whilst the grey region is the glass matrix. The parallel section (Fig. 2b) shows the strong alignment of the CNTs. The composite contained a small number of isolated pores with typical diameters of several hundred nm shown by the arrows in Fig. 2a.

3D tomography by serial FIB milling and SEM imaging was also used to study the bulk of the composite. The 3D profile of the CNTs could be reconstructed from the sequence of SEM images through the matrix (Fig. 3a and b). Either a few CNTs could be selected for detailed examination (Fig. 3a) or all of CNTs in the volume examined could be reconstructed (Fig. 
3b). The reconstructed images demonstrate that the CNTs were continuous within the glass matrix.

Figures 4a and 4b show TEM images of a FIB lift-out specimen prepared normal to the alignment direction and Figs. 4c and 4d show specimens prepared parallel to the MWCNT alignment direction. The MWCNTs can easily be distinguished from the glass matrix. Higher magnification TEM images (Fig. 4b and d) show no cracks/spaces at the MWCNT/glass interface and evidently good wetting and bonding with the matrix. All MWCNTs showed this intimate bonding with the matrix. No crystallisation of the matrix glass was evident in the area examined.

The measured densities of the preforms, ABS glass and aligned MWCNT/ABS were $0.15 \pm$ $0.04,2.25 \pm 0.03$ and $2.23 \pm 0.12 \mathrm{~g} \mathrm{~cm}^{-3}$ respectively. Using an effective density of $2.16 \mathrm{~g}$ $\mathrm{cm}^{-3}$ for the MWCNTs [6] and a volume fraction of 20\% for MWCNTs, obtained by image analysis of cross sections such as Fig. 2a, the composites are over 99\% dense. Therefore, this processing route resulted in good sintered densities with high MWCNT volume fractions ( 20\%) and with alignment of continuous MWCNTs in the matrix. It should be noted that attempts to disperse $10-15$ vol\% of randomly orientated CNTs in ceramic matrices by conventional methods have resulted in agglomeration which compromised the mechanical properties of composites [7,9].

\subsection{Micromechanical Testing}

\subsubsection{Microcantilever beams}

The microcantilever beams produced are shown in Fig. 5. The beams had lengths, widths and heights (defined in Fig. $5 \mathrm{~b} \& \mathrm{c}$ as $l, w$, and $h$ ) typically between $20-28 \mu \mathrm{m}, 4-7 \mu \mathrm{m}$ and $2-$ $5 \mu \mathrm{m}$ respectively. The beam heights were determined from a measurement taken from a $45^{\circ}$ 
tilted SEM image such as Fig. 5c corrected for the $45^{\circ}$ projection. Figure 5c also confirms that the beams were isosceles in cross-section. After final polishing at low beam currents, the free ends of the cantilever beams were clean with only a small amount of re-deposited material observed.

\subsubsection{Microcantilever bend tests}

A typical load-displacement graph for an ABS glass beam loaded to failure is shown in Fig. 6a. The ABS glass shows linear behaviour obeying Hooke's law followed by brittle fracture. There was no evidence of sub-critical crack growth, densification or plasticity as the plot remains linear until failure by fast fracture. Equations 1 and 2 were used to obtain the elastic modulus $(E)$ and the maximum tensile stress $(\sigma)$ from the load $(F)$ - displacement $(y)$ data and beam dimensions (distance from load point to built-in end, $l$, width, $w$, and height, $h) . \beta(l / w)$ in eq. 1 is a correction factor to allow for the tilting of the built-in end as described in [16] and is typically 1.3 for the beams used in this work.

$$
\begin{aligned}
& E=\frac{F}{y_{o}} \times \frac{12 l^{3}}{w h^{3}} \times \beta \\
& \sigma=\frac{36 F l}{w h^{3}}\left(\frac{1}{3} h\right)=\frac{12 F l}{w h^{2}}
\end{aligned}
$$

Applying the load-displacement data and beam dimensions to equations 1 and 2, a tensile stress-strain graph is obtained as shown in Fig. 6a (inset). The bend strength $\left(\sigma_{\text {glass }}\right)$ and elastic modulus ( $\left.E_{\text {glass }}\right)$ of glass obtained from the stress-strain graphs are presented in Table 1. The Young's modulus (77 GPa) is within reported values of 64-89 GPa [17] for bulk borosilicate glass, demonstrating the validity of the microcantilever method employed. The strength $(1.3 \mathrm{GPa})$ is much higher than it would be for bulk specimens because of the small sample size. The fracture surface of the glass beams appeared smooth except at an imperfection near to one of the tensile corners which is the likely fracture origin (Fig. 6b). 
An example of a load-displacement graph obtained from loading an aligned MWCNT/ABS composite microcantilever beam is shown in Fig. 7a. The loading region of the graph shows curvature from the onset of loading, unlike the linear elastic behaviour observed in ABS glass. Figure $7 \mathrm{~b}$ shows the response to loading and unloading of a composite microcantilever beam to successively higher displacements until failure. The composite shows a residual displacement after each cycle. The initial elastic moduli of the composite specimens, $E_{\text {comp}}$, were obtained from the tangent at the origin of a stress-strain curve (corresponding to section A on Fig. 7a). The average value was $102 \pm 3$ GPa (Table 1). This is an improvement of $c a$. $30 \%$ over that of ABS glass.

There was no significant difference between the bend strength of the composite, $\sigma_{\text {comp }}$ and that of the glass. A stable load reduction occurred just before fast fracture of the composite (Fig. 7a) in contrast to the glass (Fig. 6a). Figures 7c and d show SEM images of a beam tested to failure and a higher magnification image of the fracture respectively. The images (Fig. 7c and d) suggest the occurrence of toughening mechanisms involving bridging of the crack interfaces by the CNTs. In view of this, the load reduction before final fast fracture seen in Fig. 7a was attributed to a small amount of stable crack growth.

Table 1 Mean properties measured using ABS glass and MWCNT/ABS glass microcantilever beams. Ranges are standard error for elastic modulus and standard deviation for strength.

\begin{tabular}{|c|c|c|}
\hline & $\begin{array}{c}\text { Elastic Modulus } \\
(\mathrm{GPa})\end{array}$ & $\begin{array}{c}\text { Strength } \\
(\mathrm{GPa})\end{array}$ \\
\hline Glass & $77 \pm 3$ & $1.3 \pm 0.7$ \\
\hline Composite & $102 \pm 3$ & $1.4 \pm 0.4$ \\
\hline
\end{tabular}




\section{Discussion}

The composite Young's modulus $E_{\text {comp }}$ of $102 \mathrm{GPa}$ is $\sim 40-50 \%$ higher than previously reported for borosilicate glass matrices with $15 \%$ randomly orientated CVD MWCNT reported by Cho et al. (67 GPa) [18] and Mukhopadhyay et al. (72 GPa) [9]. The improvement can be attributed to the higher MWCNT content made possible by the present processing method, which avoids the agglomeration experienced with high MWCNT contents in previous work $[9,19]$, and to the alignment of the MWCNTs.

The elastic modulus of the MWCNTs in the composites, $E_{c n t}$, can be estimated from the composite elastic modulus using the rule of mixtures:

$$
E_{c n t}=\frac{E_{\text {comp }}-\left(1-V_{c n t}\right) E_{\text {glass }}}{V_{c n t}}
$$

where $E_{c n t}$, is the elastic modulus of the MWCNTs, $E_{\text {comp }}$ is the composite modulus, taken at the start of loading, and $E_{\text {glass }}$ is the elastic modulus of the glass matrix. The average volume fraction of MWCNTs in the composite, $V_{c n t}$ is 0.2. (Note that the beams were milled into regions free of the pores seen in Fig. 2). This gives a $E_{c n t}$ value of $200 \pm 20 \mathrm{GPa}$.

Literature values for the Young's modulus of individual CVD MWCNTs vary by over 2 orders of magnitude. Gaillard et al. [19] found $E_{c n t}$ values of ca.3, 150 and 300 GPa for precursors based on melamine, xylene and trimethylamine respectively. The variations were correlated with the defect content of the MWCNTs. The microstructures of our MWCNTs most closely resemble those of the xylene-derived MWCNTs of Gaillard et al.. Tests on individual CVD MWCNTs produced by catalytic decomposition of acetylene have also shown a wide range of elastic moduli, from as low as $30 \mathrm{GPa}$ [20] to $460 \mathrm{GPa}$ [21] with a 
corresponding variation of defect density. The present in situ measurements of MWCNTs embedded within composites show that when a statistically significant number of CVD MWCNTs is sampled, their mean Young's modulus lies towards the high end of the wide range of values reported for individual MWCNTs. Our results also show that the high temperature processing to incorporate the glass matrix neither damages the MWCNTs excessively on the one hand, nor improves the properties significantly by annealing out the defects and graphitising the MWCNTs [22] on the other.

The curvature in the load displacement plots for the composites and the hysteresis on cycling must be attributed to interwall sliding within the CNTs because it is clear from Fig. 4(b and d) that the outer walls of the CNTs are well bonded to the matrix. Detailed examination of fracture surfaces, to be reported in a future publication, also showed that the protruding sections of CNTs evident between the crack faces in Fig. 7d were formed by debonding or fracture within the CNTs in the majority of cases, rather than by fracture of the CNT-matrix interface. The interwall sliding responsible for the curvature and hysteresis in Fig. $7 \mathrm{a}$ and $\mathrm{b}$ must have occurred over a substantial proportion of the length of the beam to produce the marked reduction in composite Young's modulus close to failure (Section B of Fig. 7a). However, the fracture surfaces showed no instances of a complete inner wall of a MWCNT being pulled from the length of the beam $(\sim 20 \mu \mathrm{m})$. We conclude that sliding occurred over several micrometres around the built-in end of the beam, where the bending moment is a maximum.

Interwall sliding over such distances is consistent with the work of Kothari et al. [23] who observed pullout of $\sim 1 \mu \mathrm{m}$ long sections of the inner walls of CVD MWCNTs during fracture of amorphous silicon nitride matrix/MWCNT composite coatings. In a later work [24] Kothari et al. estimated the interwall friction in their CVD MWCNTs to be 8-20 MPa. This is 
significantly greater than the interlayer shear strength of graphite, which is $\sim 0.5 \mathrm{MPa}$ [25]. The hysteresis in the present work shows significant energy dissipation consistent with such significant interwall friction and there was evidently sufficient load transfer from one wall to another to fracture the MWCNTs throughout their cross-sections.

Like the relatively low stiffness noted above, this interwall friction can be attributed to the defective nature of the MWCNTs, e.g. by mechanical restraint from kinks in the CNT walls formed during CVD growth or by interwall bonds associated with imperfections in the $\mathrm{sp}^{2}$ hybridised wall structure. In contrast, arc-grown CNTs have a more perfect structure and therefore show higher stiffness (270-950 GPa) whilst the inner walls have been observed to pull out from the outer wall over lengths of several tens of micrometres at fracture with little apparent resistance [26].

The high bend strengths of over $1 \mathrm{GPa}$ in both glass and composites are attributed to the small flaw sizes compared with macroscopic samples. Assuming a semi-circular surface flaw and a fracture toughness of $0.7 \mathrm{MPa} \sqrt{\mathrm{m}}$ [9] for ABS glass the Griffith critical flaw size for the glass beams is $\sim 200 \mathrm{~nm}$. This is similar to the scale of the surface dimples seen in Fig. $6 \mathrm{~b}$, and examination of the fracture surface in this case suggests that fracture originated at one of the more pronounced examples of these features. The surface dimples are a result of surface etching and/or the redeposition of material during FIB milling. Ga ion implantation at the surface may also have influenced the strength although the evidence available suggests that the depth of implantation is only a few tens of nanometres [27], which is much less than the flaw size $(200 \mathrm{~nm})$.

The similarity of the bend strength of the composite to that of the ABS glass suggests a tradeoff between increased toughness in the composite due to the presence of MWCNTs on the one hand, and increased flaw size relative to the more dense and uniform ABS glass on the 
other. The stable crack growth preceding failure in Fig. 7(a), the obvious crack bridging in Fig. 7(c) and the fact that the two parts of the broken specimen remained attached after fracture by sections of MWCNT $\sim 1 \mu \mathrm{m}$ in length (Fig. 7 d-e) together demonstrate beyond doubt that the MWCNTs do indeed lead to toughening, whilst it is also obvious from Figs. 5 and 7(c)-(e) that flaws are introduced by the presence of the MWCNTs. However, the lack of an increase in stress during the stable crack growth in Fig. 7(a) also suggests that the extent of toughening, at least in the early part of the R-curve, was more limited than its spectacular appearance might suggest. We believe that one reason for this is the tapered cross section of many of the pulled out sections of MWCNT visible in Figs. 7(d) and (e): if such a section pulls out from a hole of complementary shape in the opposing surface it is evident that contact between the two, and therefore frictional traction, will be lost shortly after pullout begins.

A paper is in preparation with quantitative measurements of toughness as it develops during crack growth in notched specimens along with detailed fractography in order to develop a more quantitative understanding of these novel composites. However, the modest improvements in stiffness and fracture properties at the microscale found here, despite the high volume fraction achieved, the alignment and the continuity of the CNTs, suggest that modified CVD nanotube structures are necessary for the substantial mechanical property improvements promised by data from individual pristine CNTs to be realised in bulk ceramic matrix composites.

\section{Conclusions}

- Dense 20vol\% MWCNT/ABS glass composites in which the MWCNTs are both aligned and continuous have been fabricated. 
- Microcantilever tests showed that MWCNTs improved the elastic modulus of glass by $32 \%$ in the alignment direction. The composite had similar strength to the glass.

- Significant hysteresis and ratchetting in the load-displacement curves indicated both load transfer between the walls of the MWCNTs and substantial interwall sliding over distances of several micrometres prior to composite fracture.

- The elastic modulus of the MWCNTs in the composite was estimated to be $200 \pm 20$ GPa which is at the higher end of the wide range of values reported for individual CVD MWCNTs though still well below the stiffness of pristine, arc-grown CNTs.

- Stable crack growth prior to failure and copious amounts of crack bridging demonstrated that the MWCNTs toughen the glass.

- The elastic behaviour and interwall sliding of the CVD MWCNTs in the composite contrasts with the behaviour of individual arc-grown CNTs because of the different defect densities in the tube walls. Further development of CVD MWCNT microstructures is required to optimise composite properties.

\section{Acknowledgements}

The authors are grateful for the financial support received from The Royal Society (NG) the European Commission: the Marie Curie CONTACT Project (FP7, 238363) (NG); BNC Tubes (FP6, 33350) (NG), The European Research Council (ERC) Starting Grant (ERC2009-StG 240500 DEDIGROWTH; and ERC-2012- PoC309786 DEVICE) (NG); UK Government for Engineering and Physical Sciences Research Council (EPSRC) Pathways to Impact grants (NG) and the ERDC (Bio-inspired Composite Materials) (NG \& RIT).

\section{References}

[1] Guldi DM, Martin N, editors. Carbon Nanotubes and Related Structures. Wiley-VCH; 2010. 
[2] Balani K, Chen Y, Harimkar SP, Dahotre NB, Agarwal A. Tribological behavior of plasma-sprayed carbon nanotube-reinforced hydroxyapatite coating in physiological solution. Acta Biomaterialia. 2007;3(6):944-951.

[3] Chen Y, Zhang TH, Gan CH, Yu G. Wear studies of hydroxyapatite composite coating reinforced by carbon nanotubes. Carbon. 2007;45(5):998-1004.

[4] Otieno G, Koos AA, Dillon F, Wallwork A, Grobert N, Todd RI. Processing and properties of aligned multi-walled carbon nanotube/aluminoborosilicate glass composites made by sol-gel processing. Carbon. 2010;48(8):2212-2217.

[5] Mukhopadhyay A, Otieno G, Chu BTT, Wallwork A, Green MLH, Todd RI. Thermal and electrical properties of aluminoborosilicate glass-ceramics containing multiwalled carbon nanotubes. Scripta Materialia. 2011;65(5):408-411.

[6] Boccaccini AR, Acevedo DR, Brusatin PC, Colombo P. Borosilicate glass matrix composites containing multi-wall carbon nanotubes. Journal of European Ceramic Society. 2005;25:1515-1523.

[7] Cho J, Boccaccini AR, Shaffer MSP. Ceramic matrix composites containing carbon nanotubes. Journal of Materials Science. 2009;44(8):1934-1951.

[8] Chu BTT, Tobias G, Salzmann CG, Ballesteros B, Grobert N, Todd RI, Green MLH. Fabrication of carbon-nanotube-reinforced glass-ceramic nanocomposites by ultrasonic in situ sol-gel processing. Journal of Materials Chemistry. 2008;18:5344-5349.

[9] Mukhopadhyay A, Chu BTT, Green MLH, Todd RI. Understanding the mechanical reinforcement of uniformly dispersed multiwalled carbon nanotubes in alumino-borosilicate glass ceramic. Acta Materialia. 2010;58(7):2685-2697.

[10] Peigney A, Flahaut E, Laurent C, Chastel F, Rousset A. Aligned carbon nanotubes in ceramic-matrix nanocomposites prepared by high-temperature extrusion. Chemical Physics Letters. 2002;352(1-2):20-25.

[11] Chicatún F, Cho J, Schaab S, Brusatin G, Colombo P, Roether JA, Boccaccini AR. Carbon nanotube deposits and CNT/SiO2 composite coatings by electrophoretic deposition. Advances in Applied Ceramics. 2007;106(4):186-195.

[12] Cho J, Schaab S, Roether JA, Boccaccini AR. Nanostructured carbon nanotube/TiO2 composite coatings using electrophoretic deposition (EPD). Journal of Nanoparticles Research. 2008;10(1):99-105.

[13] Chandrashekar A, Ramachandran S, Pollack G, Lee JS, Lee G, Overzet L. Forming carbon nanotube composites by directly coating forests with inorganic materials using low pressure chemical vapor deposition. Thin Solid Films. 2008;517(2):525-530.

[14] Xia Z, Riester L, Curtin WA, Li H, Sheldon BW, Liang J, Chang B, Xu JM. Direct observation of toughening mechanisms in carbon nanotube ceramic matrix composites. Acta Mater: 52 (2004) 931-944

[15] Koos AA, Dowling M, Jurkschat K, Crossley A, Grobert N. Effect of the experimental parameters on the structure of nitrogen-doped carbon nanotubes produced by aerosol chemical vapour deposition. Carbon. 2009;47(1):30-37. 
[16] Norton AD, Falco S, Young NP, Severs J, Todd RI. Microcantilever investigation of fracture toughness and subcritical crack growth on the scale of the microstructure in $\mathrm{Al}_{2} \mathrm{O}_{3}$. Submitted to Journal of the European Ceramic Society.

[17] Bourhis E. Glass Mechanics and Technology. Wiley VCH; 2007.

[18] Cho J, Inam F, Reece MJ, Chlup Z, Dlouhy I, Shaffer MSP, Boccaccini AR. Carbon nanotubes: do they toughen brittle matrices? Journal of Materials Science. 2011;46(14):47704779 .

[19] Gaillard J, Skove M, Rao AP. Mechanical properties of chemical vapor deposition grown multiwalled carbon nanotubes. Applied Physics Letters. 2005;86(233109):1946186,13.

[20] Salvetat JP, Kulik AJ, Bonard JM, Briggs GAD, Stockli T, Metenier K, Bonnamy S, Beguin F, Burnham NA, Forro L. Elastic modulus of ordered and disoriented multiwalled carbon nanotubes. Advanced Materials. 1999;11(2):161-165.

[21] Xie S, Li W, Pan Z, Chang B, Sun L. Mechanical and physical properties on carbon nanotube. J. Phys. Chem. Sol. 2000; 61(7):1153-1158

[22] Huang W, Wang Y, Luo G, Wei F. 99.9\% purity multi-walled carbon nanotubes by vacuum high-temperature annealing. Carbon 41 (2003) 2585-2590

[23] Kothari AK, Jian K, Rankin J, Sheldon BW. Comparison between carbon nanotube and carbon nanofiber reinforcements in amorphous silicon nitride coatings. J. Am. Ceram. Soc.

91 [8] (2008) 2743-2746

[24] Kothari AK, Hu S, Xia Z, Konca E, Sheldon BW. Enhanced fracture toughness in carbon-nanotube-reinforced amorphous silicon nitride nanocomposite coatings. Acta Mater 60 (2012) 3333-3339

[25] Soule DE and Nezbeda CW. Direct basal-plane shear in single-crystal graphite. J. Appl. Phys. 39 (1968) 5122

[26] Yu MF, Lourie O, Dyer MJ, Moloni K, Kelly TF, Ruoff RS. Strength and Breaking Mechanism of Multiwalled Carbon Nanotubes Under Tensile Load. Science. 200;287(5453):637-640

[27] Kiener D, Motz C, Rester M, Jenko M, Dehm G. FIB damage of Cu and possible consequences for miniaturized mechanical tests. Materials Science and Engineering: A. 2007;459(1-2):262-272. 